University of Wollongong

Research Online

Faculty of Engineering and Information

Faculty of Engineering and Information

Sciences - Papers: Part A

Sciences

$1-1-2013$

\title{
Modelling of size effects in microforming process with consideration of grained heterogeneity
}

H N. Lu

University of Wollongong, haina@uow.edu.au

D B. Wei

University of Wollongong, dwei@uow.edu.au

Z Y. Jiang

University of Wollongong, jiang@uow.edu.au

$\mathrm{X} \mathrm{H.} \mathrm{Liu}$

Northeastern University

K Manabe

Tokyo Metropolitan University

Follow this and additional works at: https://ro.uow.edu.au/eispapers

Part of the Engineering Commons, and the Science and Technology Studies Commons

Research Online is the open access institutional repository for the University of Wollongong. For further information contact the UOW Library: research-pubs@uow.edu.au 


\title{
Modelling of size effects in microforming process with consideration of grained heterogeneity
}

\begin{abstract}
Size effect is a special phenomenon in metal micro-forming process. As the deformation process is scale down to micro/mesoscale, the characteristics of single grain involved in the deformed region play a significant role on the material mechanical behaviours resulting in the invalidation of classical theories in microforming. This paper presents a newly developed material model in microscale on the basis of the grained heterogeneity (e.g. grain size, shape and deformability) and specimen dimension. Voronoi tessellation has been employed to describe the polycrystalline aggregate. The grain shape is controlled by the centroidal-voronoi algorithm to drive grains into steady state. Hardness of the grains obtained from Nano-indentation is used to identify the scatter of the grained deformability. Applying the new material model, the micro-compression test of pure copper is numerically simulated by finite element method (FEM). The influences of grain size and feature size on the deformation behaviours are discussed. The numerical simulation results are in good agreement with the experimental results in terms of the flow stress curves and profile of deformed parts. Based on the novel material model, a FE model of microcross wedge rolling is established and the obtained results show the strain of specimen core region increases with the magnification of grain size. (C) 2013 Elsevier B.V. All rights reserved.
\end{abstract}

\section{Keywords}

grained, heterogeneity, microforming, process, modelling, consideration, effects, size

Disciplines

Engineering | Science and Technology Studies

\section{Publication Details}

Lu, H. N., Wei, D. B., Jiang, Z. Y., Liu, X. H. \& Manabe, K. (2013). Modelling of size effects in microforming process with consideration of grained heterogeneity. Computational Materials Science, 77 44-52.

This journal article is available at Research Online: https://ro.uow.edu.au/eispapers/1197 


\title{
Modelling of Size Effects in Microforming Process with Consideration of Grained Heterogeneity
}

\author{
H.N. Lu ${ }^{1)^{*}}$, D.B. Wei ${ }^{1)}$, Z.Y. Jiang ${ }^{1)}$, X.H. Liu' ${ }^{2)}$, K. Manabe ${ }^{3)}$ \\ ${ }^{1)}$ School of Mechanical Materials and Mechatronic Engineering, University of Wollongong, Wollongong, Australia; ${ }^{2)}$ State Key Laboratory \\ of Rolling and Automation, Northeastern University, Shenyang, China; ${ }^{3)}$ Department of Mechanical Engineering, Tokyo Metropolitan \\ University, Tokyo, Japan
}

* Corresponding author: E-mail address: h1418@uowmail.edu.au; Tel.: +61 433383697

\begin{abstract}
Size effect is a special phenomenon in metal micro-forming process. As the deformation process is scale down to micro/meso scale, the characteristics of single grain involved in the deformed region play a significant role on the material mechanical behaviours resulting in the invalidation of classical theories in microforming. This paper presents a newly developed material model in micro scale on the basis of the grained heterogeneity (e.g. grain size, shape and deformability) and specimen dimension. Voronoi tessellation has been employed to describe the polycrystalline aggregate. The grain shape is controlled by the centroidal-voronoi algorithm to drive grains into steady state. Hardness of the grains obtained from Nano-indentation is used to identify the scatter of the grained deformability. Applying the new material model, the micro-compression test of pure copper is numerically simulated by finite element method (FEM). The influences of grain size and feature size on the deformation behaviours are discussed. The numerical simulation results are in good agreement with the experimental results in terms of the flow stress curves and profile of deformed parts. Based on the novel material model, a FE model of micro cross wedge rolling is established and the obtained results show the strain of specimen core region increases with the magnification of grain size.
\end{abstract}

Keywords: Microforming, Size effect, Grained heterogeneity, Constitutive model

\section{Introduction}

In recent years, micro system technology, a prominent technology to fabricate microparts, is attracting more and more attention due to the increasing demand on micro-scale products. The metal forming in conventional scale has been developed for hundreds of years and previous researchers have established a series of classic theories for macro-scale deformation, however, many of which do not work when the metal deformation scales down to a certain geometrical scale [1-3] due to size effect. The most common phenomena of size effect associated with miniaturisation are related to the deformation behaviour which is normally described by flow stress model [4].

Many explorations on this issue were conducted using both experimental and numerical methods and the corresponding constitutive models were developed as well. Hall [5] and Petch [6] studied the effect of grain size on the yield stress and found a linear relationship between the yield stress and the reciprocal of the square root of grain size. Miyazaki et al [7] investigated the effect of specimen size on the flow stress of rod specimen of polycrystalline $\mathrm{Cu}-\mathrm{Al}$ alloy and an affect zone model is proposed to explain why the flow stress decreases with the dimensional reduction of specimen. Leu [8] established a flow stress model as a function of the ratio of the sheet thickness to grain size based on the affect zone model, the pile-up theory of dislocation and Hall-Petch relationship.

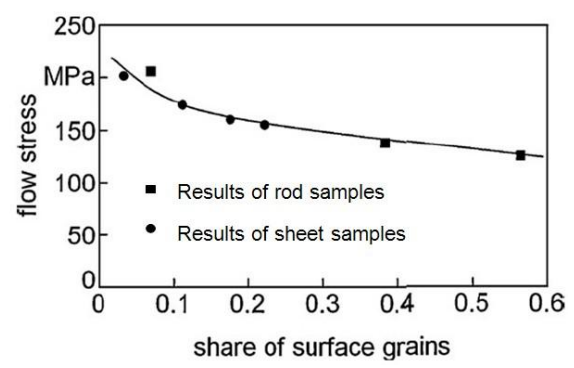

Figure 1. Change of flow stress with increasing share of surface grains [10]

Geiger and Engle [9] proposed the surface layer model, which divided the specimen into two portions, inner and surface layer portions, to explain the reduction of flow stress in metal microforming process. A systematic influence of the sample size was proposed by Kals et al. [10] by defining a quantitative value for the share of surface grains. The influence of the surface grain was shown in Fig. 1. Lai et al [11] developed a mixed model 
by combining the modified Hall-Petch relation and surface layer model. Based on the composite and surface layer models, Liu [12] developed a constitutive model considering the grain boundary strain strengthening.

The grained heterogeneity was taken into account by Chan et al. [13] to investigate the decrease of flow stress and scatter of grained behaviour due to different grain size, shape and orientation. The grained heterogeneity was identified using nanoindentation test by the authors to simulate the micro cross wedge rolling process and an inhomogeneity coefficient has been introduced [14].

The voronoi tessellation was introduced to generate polycrystalline aggregate using probability theory. This method subdivides a space tessellation into voronoi polyhedral, which is similar to morphogenetic process of nucleation and growth from random seeds [15]. The concept of the voronoi polygon has been used extensively in material science, especially for modelling random microstructure of grain aggregates [16]. Zhang and Tong [17] presented the numerical computation of 3D as a general investigation tool that featured an aggregate computation including element type and geometrical representation of grain boundary. Based on voronoi tessellation, lognormal and gamma distribution were used to describe the distribution of grain size in polycrystalline by Zhu et al. [18] and Dalla et al. [19].

The main objective of this study is to introduce a constitutive material model based on the surface layer model and intragranular heterogeneities that involves the difference between the grain interior and boundaries and the scatter of deformation behaviour among grains. The 3D aggregate of polycrystalline is represented by a voronoi tessellation. Applying the novel material model and 3D full scale polycrystalline structure, the microcompression test of pure copper is numerically simulated by finite element method (FEM). In order to validate the accuracy of the presented material model, physical experiments are conducted to compare the simulation results with experimental ones. The scatter of grained heterogeneity is analysed in terms of flow stress and free surface asperity of the deformed part. A FE model of micro cross wedge rolling is established based on the new material model and the influence of grained heterogeneity on strain distribution is discussed briefly.

\section{Scatter of constitutive model in microforming process}

The most common used parameter to describe the behaviour is the flow stress curve. In this paper, a mixed material model based on modified Hall-Peth relationship, surface layer model and grained heterogeneity is proposed.

\section{1. hall-peth relationship}

The effect of grain size on flow stress is an important aspect of polycrystalline metal plastic deformation. The Hall-Petch Equation is the most widely accepted empirical theory describing the effect of grain size on the yield stress and a linear relationship between the yield stress and reciprocal of the square root of the grain size was found $[5,6]$. It was further developed based on the dislocation pileup theory as Equation 1 described.

$$
\sigma=M \tau=M\left(\tau_{i}+K d^{-1 / 2}\right)
$$

where $M$ is a relevant factor to grain orientation and it is 3.1 for polycrystal according to Taylor Theory, $\tau$ is shear stress, $K$ is a constant, $d$ represents the grain size [20].

\subsection{Composited model}

The methodology of studying grain size effect on flow stress is based on the composite model developed by Kocks [21] and Meyers and Ashworth [22]. This model has been proven to be a valid and effective approach to describe the relationship between the material yield stress and grain size. This composite model supposes that the deformation polycrystalline is composed of a hard grain boundary region and a soft grain interior region, as shown in Fig. 2.

A composite model is thus proposed to calculate the flow stress of polycrystalline aggregate in the following:

$$
\sigma_{s}=(1-f) \sigma_{i}+f \sigma_{b}
$$

where $\sigma_{s}$ is the flow stress of polycrystalline aggregate. $f$ is the volume fraction of grain boundary. $\sigma_{i}$ and $\sigma_{b}$ are the flow stresses of grain interior and boundary respectively.

Assuming that the grain size is $D_{g}$ and the thickness of grain boundary layer is $t_{g}$. The relationship between the thickness of grain boundary and the grain size is described as the following equation [23].

$$
t_{g}=k D_{g}^{n}(0<n<1)
$$

where $k$ and $n$ are regarded as constants for a specific material. According to the previous research report [24], $k$ and $n$ are determined to be 0.133 and 0.7 respectively when the material is copper. 
In this study, an equivalent sphere (Fig. 2) is proposed to take the place of the voronoi cell when the grain size is calculated. The volume of sphere $V_{e s}$ is equal to that of the voronoi cell $V_{v c}$ and the grain size is equal to the diameter of the equivalent sphere. Therefore,

$$
V_{e s}=V_{v c}=\frac{4}{3} \pi\left(\frac{D_{g}}{2}\right)^{3}
$$

3D simplex integration method is used to calculate the volume of each voronoi cell. Assuming there are $m$ grains involved in the inner portion of specimen, the volume faction of grain $i(i=1,2, \ldots, m)$ in the inner portion of specimen is given just below:

$$
v_{c}(i)=\frac{4\left(D_{g}(i) / 2\right)^{3}}{3\left(r_{s}-D_{g m}\right)^{2}\left(h_{s}-2 D_{g m}\right)}
$$

where $D_{g m}$ is the average grain size and $D_{g}(i)=\left(\frac{6 V_{v c}(i)}{\pi}\right)^{\frac{1}{3}} \cdot r_{s}$ and $h_{s}$ are the radius and height of the specimen respectively as shown in Fig. 3

Substituting Equations 3 to 4, the volume faction of the boundary of grain $i$ can be expressed as:

$$
f_{c}(i)=1-\frac{V_{\text {interior }}(i)}{V_{v c}(i)}=\left(\frac{2 t_{g}(i)}{D_{g}(i)}-1\right)^{3}+1=\left(2 k D_{g}^{n-1}(i)-1\right)^{3}+1
$$

where $f_{c}(i)$ is the volume faction of the boundary of grain and $V_{\text {interior }}(i)$ is the volume of grain interior. The total volume fraction of the grain boundaries is obtained,

$$
f=\sum_{i=1}^{m} v_{c}(i) \times f_{c}(i)=\sum_{i=1}^{m} \frac{4\left(D_{g}(i) / 2\right)^{3}\left(\left(2 k D_{g}^{n-1}(i)-1\right)^{3}+1\right)}{3\left(r_{s}-D_{g m}\right)^{2}\left(h_{s}-2 D_{g m}\right)}
$$

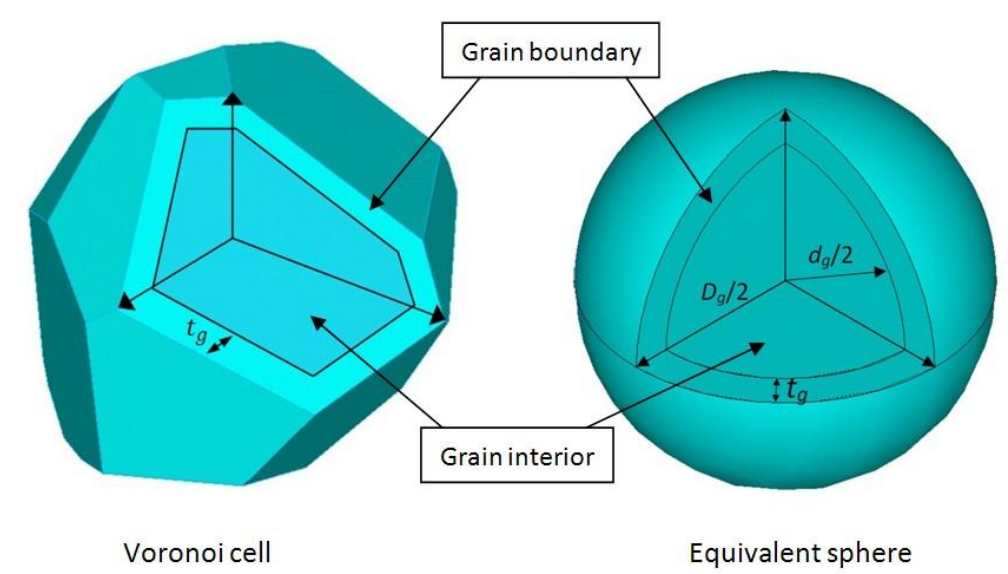

Figure 2. Grain interior and grain boundary model

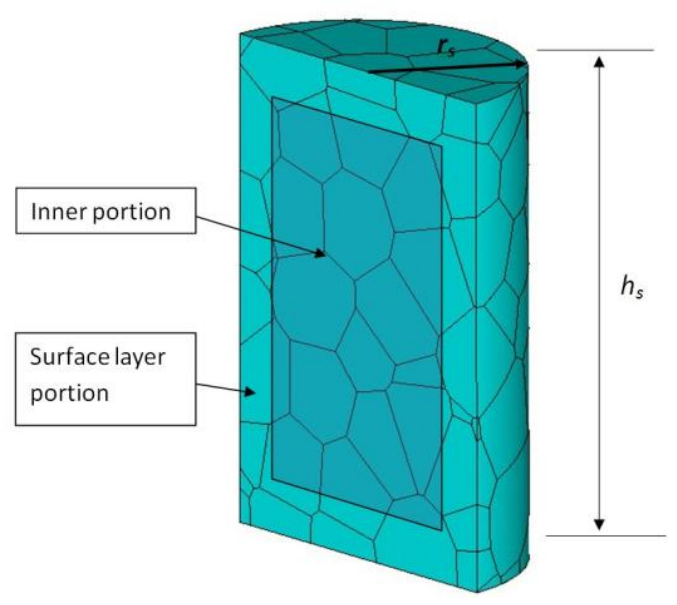

Figure 3. Surface layer and inner portion of a cylindrical spencimen 


\subsection{Surface layer model}

The surface layer model is utilised to explain the geometry size effect on the decrease of flow stress during miniaturisation. The specimen is subdivided into two portions: surface layer and inner part as shown in Fig.3. The flow stress of the material is composited by that of inner portion and surface layer of specimen as follows:

$$
\sigma=\gamma \sigma_{\text {inner }}+(1-\gamma) \sigma_{\text {surf }}
$$

where $\sigma$ is the flow stress of material. $\sigma_{\text {inner }}$ and $\sigma_{\text {surf }}$ are the flow stress of the grain located at inner and surface region, respectively. $\gamma$ is the fraction of inner portion and it can be expressed as follows:

$$
\gamma=\frac{\left(r_{s}-D_{g m}\right)^{2}\left(h_{S}-2 D_{g m}\right)}{r_{s}^{2} h_{s}}
$$

The flow stress of inner portion can be described by that of polycrystalline aggregate, $\sigma_{\text {inner }}=\sigma_{s}$, the grains located at the free surface have less hardening effect than that of inner grains. Therefore, the grain boundary strengthening effect in surface layer is ignored. The flow stress of surface layer portion is equal to that of grain interior, $\sigma_{\text {surf }}=\sigma_{i}[12]$. As a result, the geometry size effect is taken into account in the composite model. The flow stress in microfoming can be expressed:

$$
\sigma=(1-\gamma f) \sigma_{i}+\gamma f \sigma_{b}
$$

A parameter $\mathrm{F}$ is introduced to be on the half of $\gamma f$,

$$
F=\gamma f=\frac{4}{3 r_{s}^{2} h_{s}} \sum_{i=1}^{m}\left(D_{g}(i) / 2\right)^{3}\left(\left(2 k D_{g}^{n-1}(i)-1\right)^{3}+1\right)
$$

Then, Equation 9 can be rewritten as:

$$
\sigma=(1-F) \sigma_{i}+F \sigma_{b}
$$

where $F$ is defined as a size-effect factor and can be figured out when the dimension of micro part and its grain size are specified. Therefore, in the developed constitutive model, the flow stress of grain interior and grain boundary are unknowns. No less than two strain-stress curves of deformed material with different grain sizes are required to determine $\sigma_{i}$ and $\sigma_{b}$. The values of $\sigma_{i}$ and $\sigma_{b}$ at a certain strain can be obtained. The flow stress curves of grain interior and boundary are finally determined. Based on $\sigma_{i}$ and $\sigma_{b}$, the flow stress curve of the corresponding material with an arbitrary grain size can be figured out. When the dimension of cylindrical sample is $\Phi 0.8 \mathrm{~mm} \times 1.2 \mathrm{~mm}$, a series of flow stress curves with different grain sizes are obtained based on Equation11 as shown in Fig. 4.

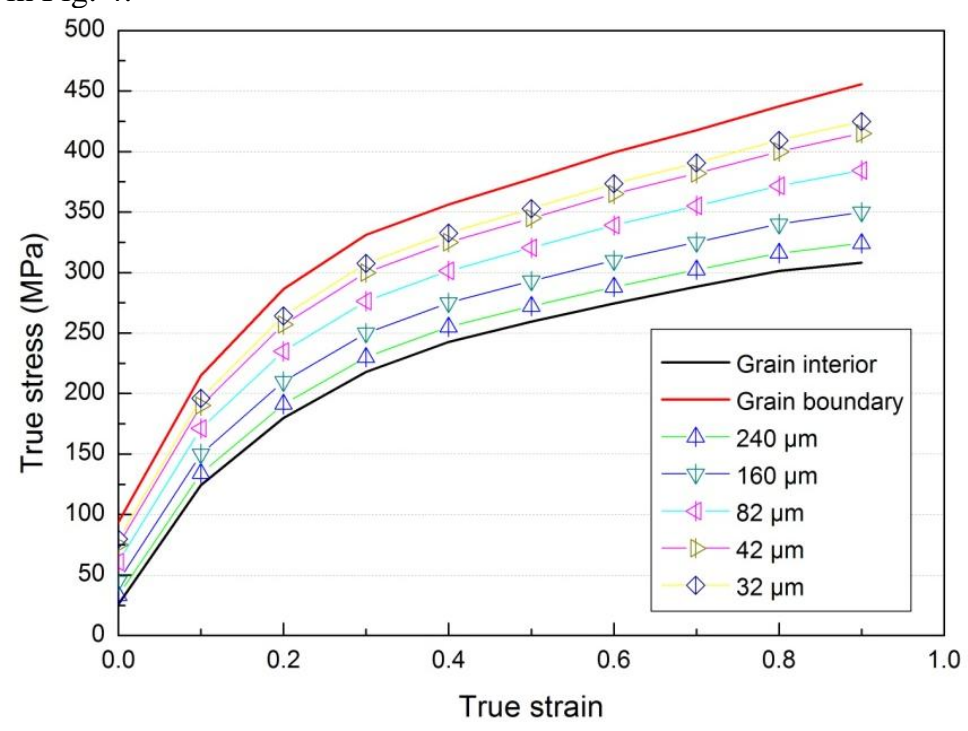

Figure 4. Strain-stress curves determined according to Equation 11

\subsection{Grained heterogeneity}

Actually, when the specimen size is scaled down to micro scale, deformation behaviour is characterised by the deformation regions with only a few grains. The distributions of different grains with different orientations, sizes 
and shapes have a significant influence on the size effect that causes inhomogeneous deformation behaviour and the scattering of material properties.

In this study, nanoindentation was adopted to indentify the grained heterogeneity. Hardness is the ability of a material to resist plastic deformation via scratch or indentation. Hardness of grains obtained from nanoindentation test (Fig. 5) is an effective parameter to estimate the deformability of individual grain inside the micro part. According to the grain hardness, grained heterogeneity can be figured out. Statistical distribution of hardness is divided into 7 classes (Fig. 6a). The ratio of the hardness at each class divided to the average hardness is defined as an inhomogeneity coefficient to make the strain-stress curves vary following the statistical distribution. As demonstrated in Fig.6b, $\alpha$ is the inhomogeneity coefficient.

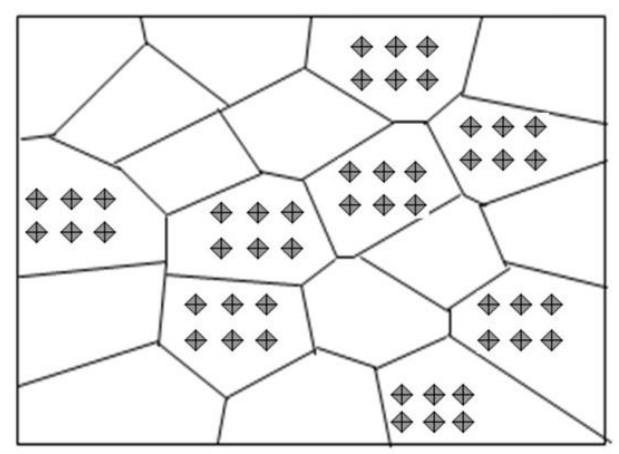

Figure 5. Illustration of nanoindentation
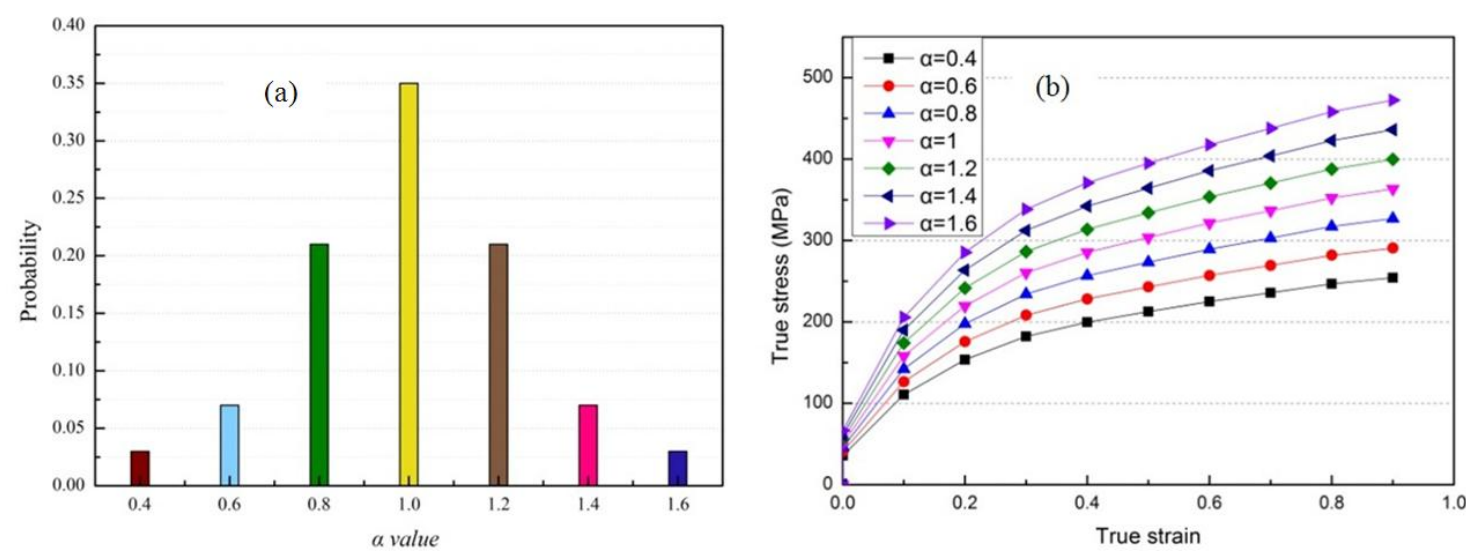

Figure 6. Strain-stress curves based on scatter of grained deformation behaviour. (a) statistical distribution of $\alpha$ value, (b) flow stress of inhomogenous material

In order to have a deep understanding on the deformation behaviour in micro-compression, grains are meshed and $3 \mathrm{D}$ fully aggregates are computed with a FE method. The material constitutive model illustrated in Section 2 is to attribute to the specific region, viz. individual grain randomly.

The deformed part is represented based on a space tessellation into 3D voronoi diagram which can describe grain boundary and generation process of grain vividly.

Voronoi diagram can be defined as $n$ distinct regions $V_{i}$ based on an open set $\Omega$ and $n$ different seeds $z_{i}(i=0$, $1, \ldots, n-1)$ such as:

$$
V_{i}=\left\{w \in \Omega \mid d\left(w, z_{i}\right)<d\left(w, z_{j}\right) j=0,1, \ldots, n-1, j \neq i\right\}
$$

where $d$ is a function of distance. Voronoi polyhedra are the influence zones of these seeds that are their mass centre.

A 3D domain is decomposed into a certain number of subdomains. Then, the seeds are spread into these subdomains. Assuming the centre of a cube is $(x, y, z)$, the seed will lie $\lambda \delta a / 2$ away from the central point. Exactly, the coordinate of the seed position should be $(x+\lambda \delta a / 2, y+\lambda \delta a / 2, z+\lambda \delta a / 2) . \delta$ is the maximum distance between the seed and the cubic centre, and $\lambda$ is a random number between -1 and 1 . Both $\delta$ and $\lambda$ are so called shape factors which will determine the shapes of Voronoi cells. A critical distance $d$ between different seeds is defined to prevent each seed from being excessively close to one another. After that, 3D Voronoi polyhedra are generated by taking these seeds as the generating points. The grain radius $D_{g}$ is defined as the cubic root of the 
grain volume and the average grain radius $D_{g m}$ is defined as the cubic root of average grain volume within the 3D grain.
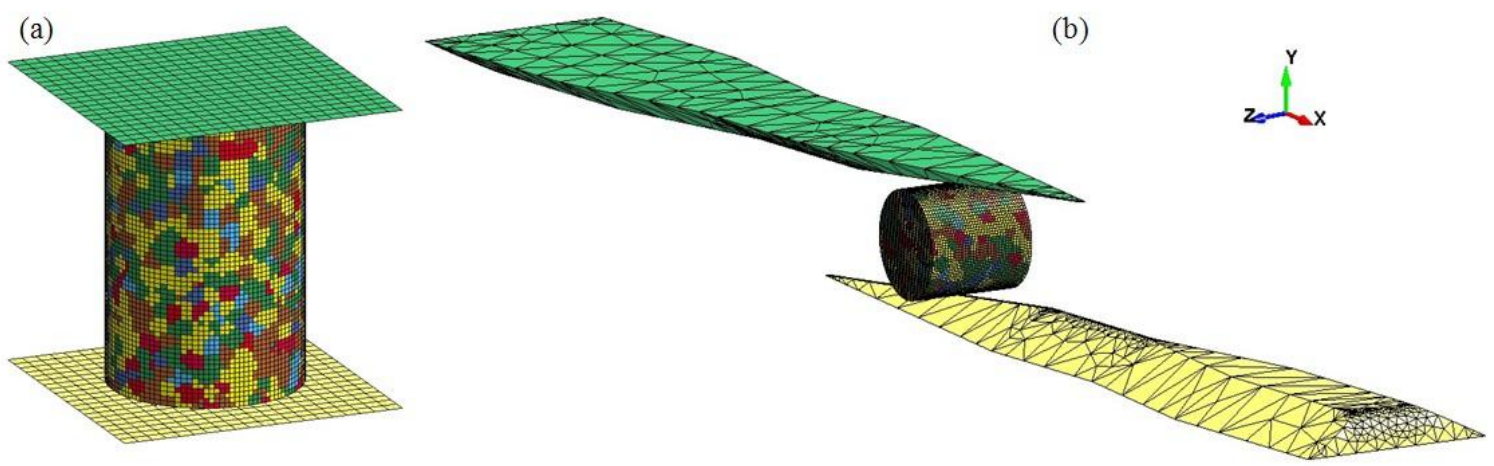

Figure 7. FEM simulation models: (a) micro compression model and (b) MCWR model

After generating the appropriate topological structure of grains within the specimen, this geometrical feature should be imported to commercial FEM software ANSYS/LS-DYNA and meshed into elements. The newly developed material model is implemented considering grained heterogeneity. Fig. 7 shows the FE models of micro compression and MCWR processes. In the micro compression model, a cylindrical workpiece of $\Phi 0.8 \times$ $1.2 \mathrm{~mm}$ is meshed using eight-noded structural Solid Element 164 which owns six degrees of freedom per node, translation and rotation in $\mathrm{x}, \mathrm{y}$ and $\mathrm{z}$. One point integration and viscous hourglass control were employed for all the elements for reducing computation time and ensure stability during large deformation. Two plate tools are meshed using four-noded thin shell element 163 . The upper plate is fixed in $\mathrm{x}, \mathrm{z}$ directions and the lower one is constrained in all directions. A velocity of $0.01 \mathrm{~mm} / \mathrm{s}$ in y direction is applied on the upper plate. The friction coefficient between tools and specimen was set as 0.03 . For each grain size, 10 different polycrystalline aggregates of workpiece were generated stochastically by the algorithm of 3D voronoi tessellation. In MCWR model, a cylindrical workpiece and two flat wedges are meshed using Solid Element 164 which owns the same attributes with that of workpiece in compression model.

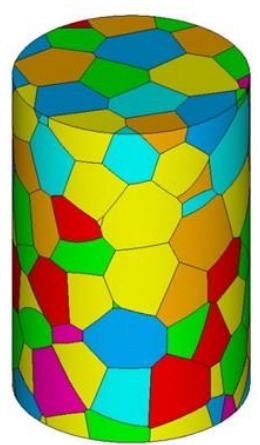

(a)

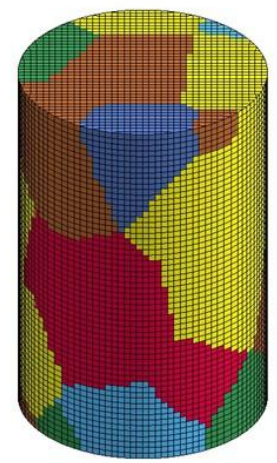

(d)

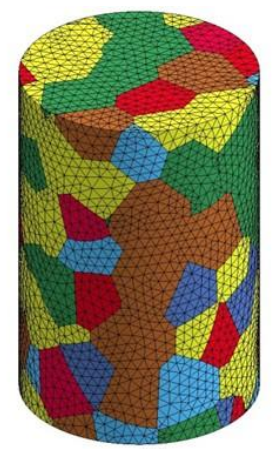

(b)

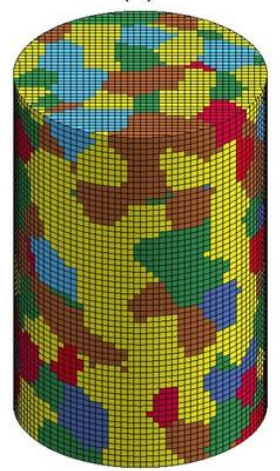

(e)

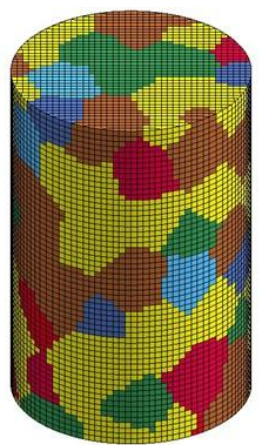

(c)

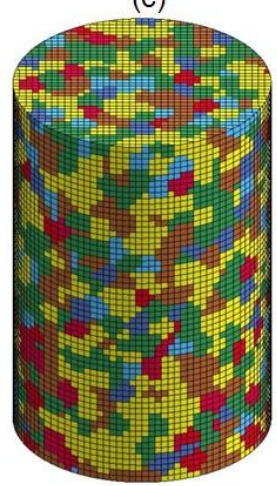

(f)

Figure 8. Aggregate of polycrystalline, (a) tessellation in 140 vornoi polyhedral. (b) freely meshed model with 140 subdomains, $(\mathrm{c}, \mathrm{d}, \mathrm{e}, \mathrm{f})$ Microstructure mapping on a $1313 \times 60$ cubic element mesh and domain with subdomain numbers 140, 26, 385, 2520 respectively. 
During meshing process, space is discretised and described with a 3D digital image composed of voxels (pixels in 3D) that can be labelled with the number of grains that they belong to. According to the characteristics of voronoi diagram, a distance function is introduced and a segmentation algorithm associates each voxel to the nearest nucleus. Fig. 8a gives an example with an aggregate composed of 140 grains. This image can be discretised into finite elements using a regular mesh or free mesh as shown in Fig. 8b and 8c respectively. Fig. $8 \mathrm{~b}$ shows the FE model meshed freely with tetrahedral elements and Fig. 8c - $\mathrm{f}$ illustrates the aggregate of grains discretised into regular cubic elements. In Fig. 8c - f, the samples containing 140, 26, 385, 2520 grains were discretised into $1313 \times 60$ elements.

Mechanical fields can be quite sensitive to the element type and especially to the geometrical description of grain boundaries and mesh type. Although free meshing can describe accurately at the vicinity of the grain boundaries where the large stress and strain gradient locate, it may generate abnormal elements and increase computational errors. In addition, tetrahedral elements are not competent to simulate plastic deformation because of its rigidity. In order to eliminate the effects of meshing, the specimen is meshed with regular cubic elements as an alternative. However, the regular meshing cannot describe grain boundary accurately even using large number of elements. Therefore, suitable meshing density should be specified to control the numerical simulation cost and geometrical error of grain boundaries synchronously.

\section{Establishment of experiment}

\subsection{Specimen preparation}

The size effect was investigated using compression test of pure copper. The dimensions of cold drawn samples are $\Phi 0.8 \mathrm{~mm} \times 1.2 \mathrm{~mm}$. The samples were annealed at the temperature $700{ }^{\circ} \mathrm{C}$ and the holding times were $0.1,0.5$, 2 and 8 hours respectively in order to produce various grain sizes. Argon was used to prevent copper samples from oxidation. Some samples were etched in a solution of $5 \mathrm{~g} \mathrm{of} \mathrm{FeCl}_{3}, 50 \mathrm{ml}$ of $\mathrm{HCl}$ and $100 \mathrm{ml} \mathrm{H}_{2} \mathrm{O}$ for 10 $15 \mathrm{~s}$. The microstructures of tested samples were obtained using optical microscope as shown in Table 1. The grain size increases with the holding time.

The relationship between the specimen geometrical dimension and material grain size is taken into account to investigate the size effect during deformation by the ratio of the specimen size ( $\mathrm{g}$ ) to grain size (s) as shown in Equation 14.

$$
\lambda=\frac{\text { specimen radius }(g)}{\text { grain size }(s)}
$$

\subsection{Compression test}

Compression test is usually used to investigate the flow stress and other deformation behaviours for large deformation. In this research, the compression test was conducted at room temperature by employing the desktop servo-press machine. The punch runs at a constant speed of $0.01 \mathrm{~mm} / \mathrm{s}$. The contact surface between the tool and sample are lubricated in order to reduce the influence of friction. The reduction was $60 \%$. Fig. 8 shows the load-displacement curves obtained from both experiments and simulations, which indicates a quite small discrepancy between them.

Table 1. Microstructure of specimens and grain size before and after compression

\begin{tabular}{|c|c|c|c|c|}
\hline Holding time $(\mathrm{h})$ & 0.1 & 0.5 & 2 & 8 \\
\hline Grain size $(\mu \mathrm{m})$ & 59 & 125 & 240 & 470 \\
\hline$\lambda$ & 7.5 & 3 & 1.6 & 0.85 \\
\hline Microstructures & & & & 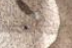 \\
\hline
\end{tabular}

\section{Analysis}

In microforming process, grain heterogeneity plays an important role in flow stress and surface quality. 


\subsection{Flow stress}

As shown in Fig. 9, the punch load increases gradually when $\lambda$ increases from 0.85 to 7.5 . It agrees with the feature that the increase of $\lambda$ value will cause an increase of flow stress in a certain range of the ratio of the specimen size to grain size [25].

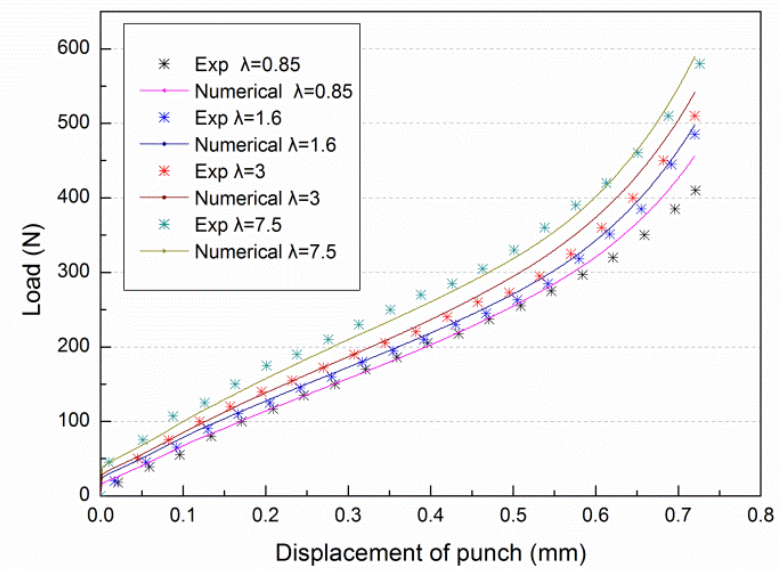

Figure 9. Punch force vs displacement
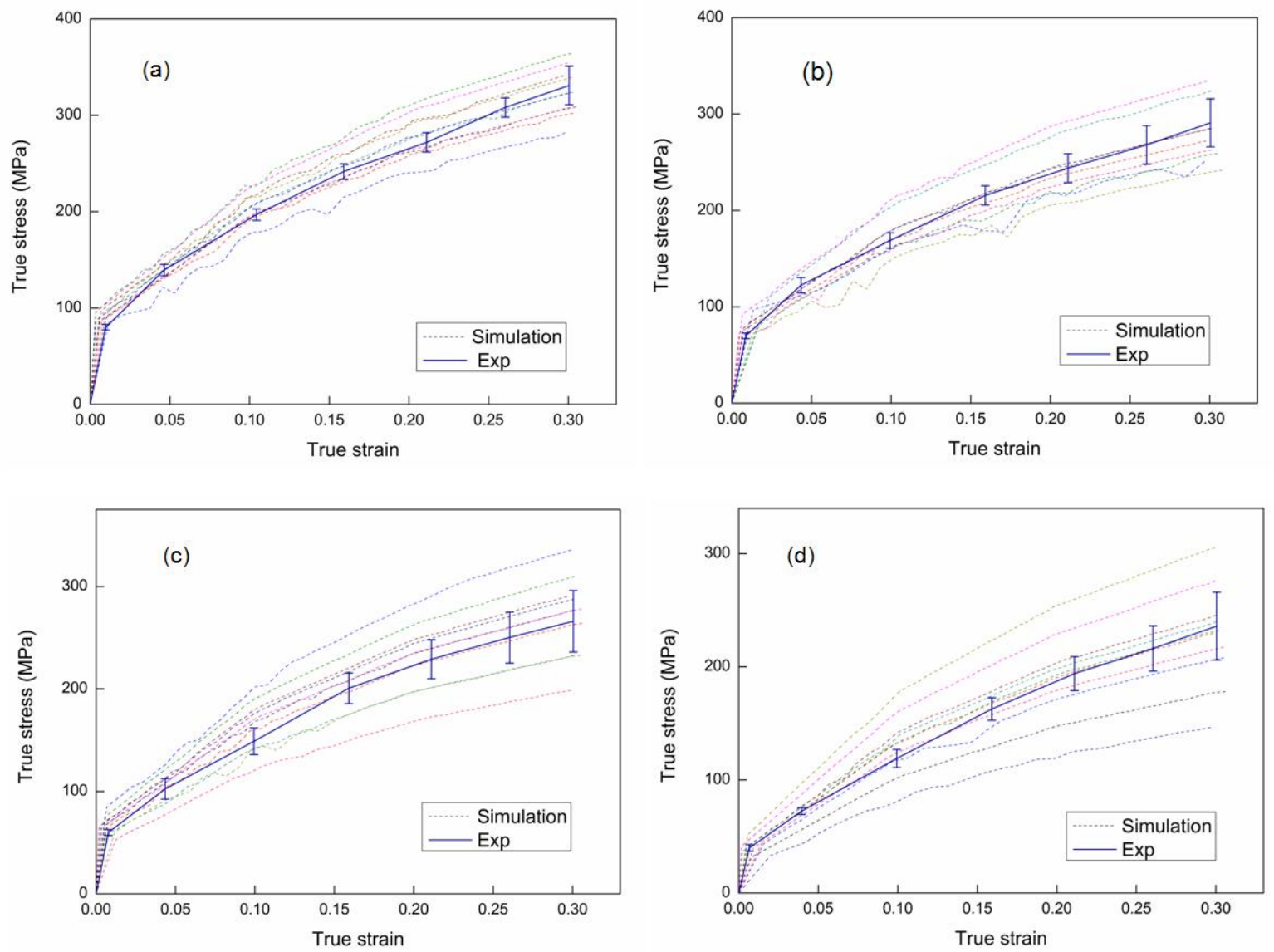

Figure 10. Comparison of stress-strain curves obtained from experiment and simulation (a) $\lambda=7.5$, (b) $\lambda=3$, (c) $\lambda=1.6$, (d) $\lambda=0.85$

Both the experimental and numerical flow stresses decrease significantly as $\lambda$ decreases as shown in Fig. 10. The four experimental stress values with corresponding strain of 0.3 are 330.9, 290.4, 265.7 and 235.7 MPa respectively which decrease significantly. The 10 numerical strain-stress curves obtained distribute dispersedly due to material heterogeneity and the scatter increases with the increasing grain size because a single grain plays a more significant role on material behaviour when the amount of grains decreases. According to Equation 11, the effects of the grain interior and boundary are integrated as a single body, so the volume fractions of grain 
boundary and surface layer grains vary while $\lambda$ changes from 0.85 to 7.5 . Consequently, grain boundary strengthening effect is enhanced and soften effect of surface layer grains is lessened due to the increase of $\lambda$, which accounts for the change of the mean flow stress obtained in the FE simulation and experimental results.

Fig. 10 also shows the error of flow stress becomes smaller with the increase of $\lambda$. The scatter of numerical flow stress is more significant than that of compression experiment and the scatter of the simulation with $\lambda$ increasing from 0.85 to 7.5 . It can be seen that the scatter becomes less significant with an increase of $\lambda$, which means that the effect of individual grain on the deformation decreases with an increase of $\lambda$.

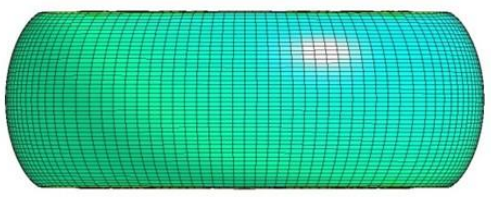

(a)

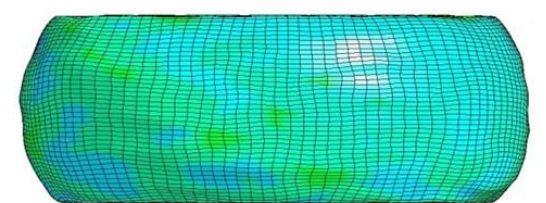

(b)

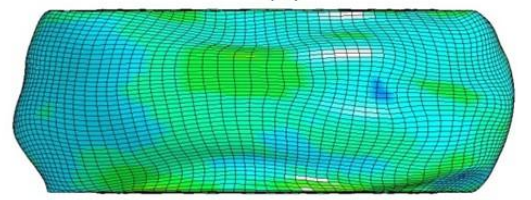

(d)

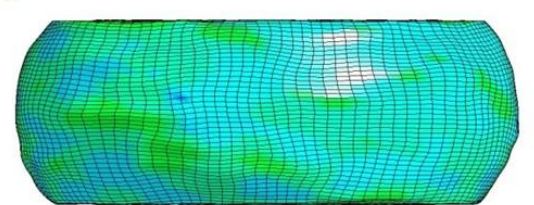

(c)

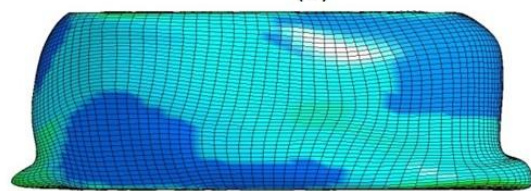

(e)

Figure 11. Profile of specimens after compression, (a) homogenous model with $\lambda=1.6$, (b) $\lambda=7.5$, (c) $\lambda=3$, (d) $\lambda=1.6$, (e) $\lambda=0.85$

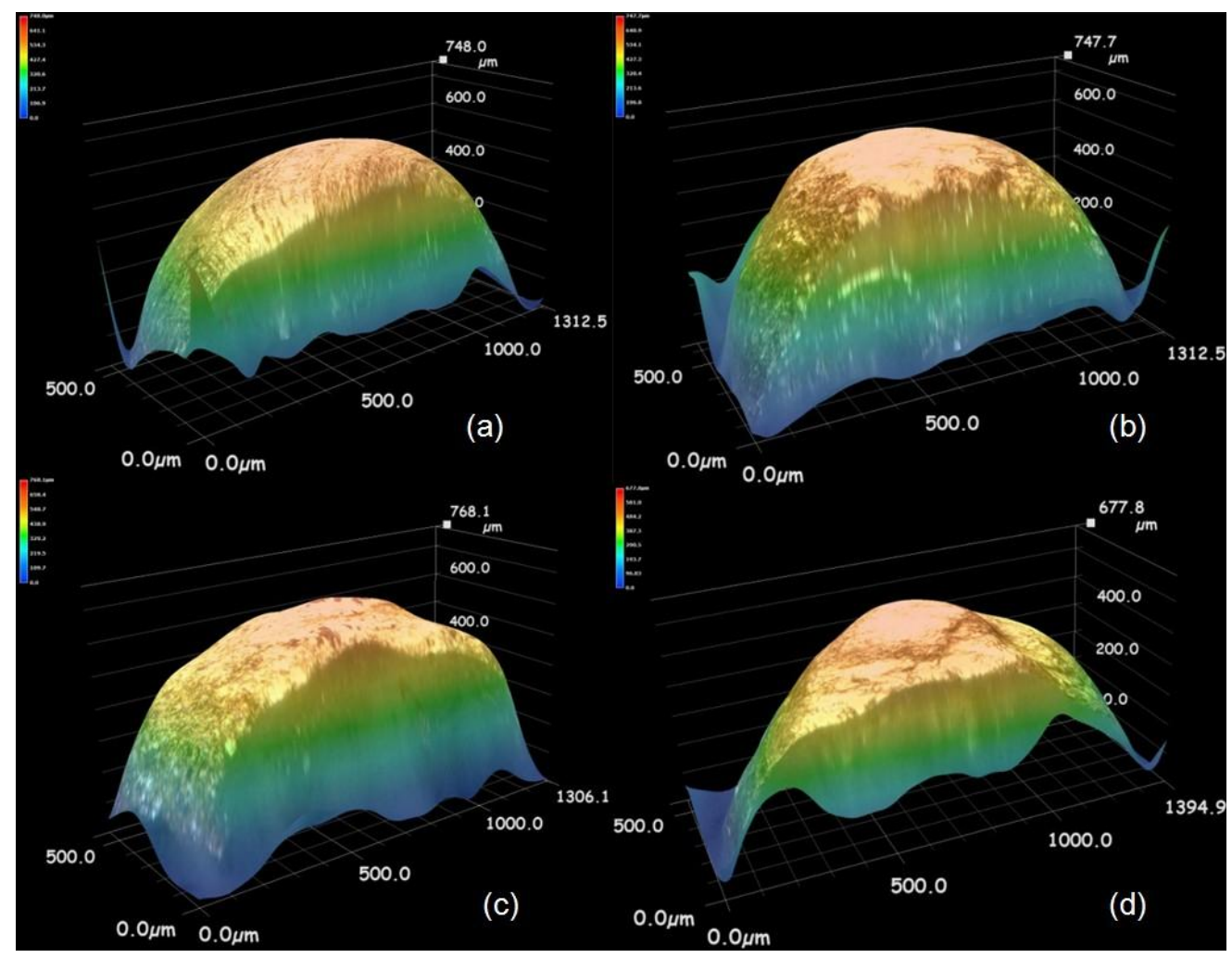

Figure 12. Profile of specimens with various grain sizes in compression test, (a) $\lambda=7.5$, (b) $\lambda=3$, (c) $\lambda=1.6$, (d) $\lambda=0.85$ 


\subsection{Surface asperity}

The scatter of individual grained plastic deformability affects the specimen profile as well. Consequently, it supplies another method to penetrate size effect on the deformation behaviour and quality of micro product. Side surface asperity of specimen after compression has been measured in simulations and experiments.

When there are only a few grains involved in the deformation, deformation incompatibility between grains cannot be accommodated by enough strain gradient of neighbouring grains. This inhomogenous deformation will influence the surface asperity of micro deformed part. Fig. 11 shows the surface profile in the simulation with $\lambda$ ranging from 0.85 to 7.5 and model with homogenous material constitutive properties. Based on the simulated contours of side surface of the sample model, it can be seen that size effect on the increasing inhomogeneous deformation behaviour with the decrease of $\lambda$ can be obtained by simulations. It reveals that the side surface become rougher with the decrease of $\lambda$, while the specimen size keep constant. The half surface profile of the compressed sample in experiment was composited by 3D digital microscope as shown in Fig. 12. It reveals that the side surfaces become rougher when $\lambda$ decreases, which has a good agreement with the simulation results.

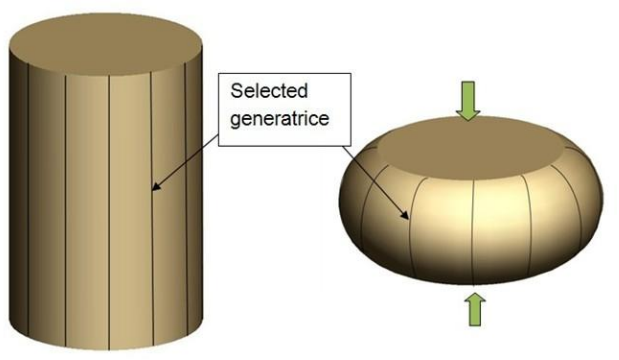

(a)

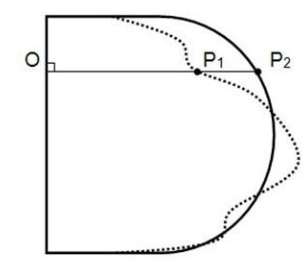

(b)

Figure 13. Illustration of the definition of $H_{s a}$, (a) generatrices for measuring, (b) radial difference between points on selected and reference generatrices

In order to measure the surface asperity quantitatively, twelve generatrices uniformly spreading on the side surface of cylindrical specimen were determined as illustrated in Fig. 13a. After deformation, surface becomes roughed comparing with the original sample surface. Assuming the deformation behaviours of grains inside the sample are homogenous, side surface of the compressed sample is perfectly smooth. One generatrice of this assumed compressed sample is used as a reference for both the experimental and numerical analyses. In the experiment, the generatrice profiles on the compressed samples were measured by 3D digital microscope and the coordinates of 20 points located evenly on each generatrice were selected to estimate the surface asperity.

The absolute difference in radial direction between point $\mathrm{P}_{1}$ the selected generatrices and point $\mathrm{P}_{2}$ on the reference generatrice at the same height (Fig. 13b) is figured out. The meaning value of the absolute differences obtained from all of the twelve generatrices is defined as $H_{s a}$ and has been used to assess the surface roughening. The experimental and numerical results are compared as shown in Fig. 14. It can be seen that the surface is rougher when $\lambda$ value decreases, which means the effect of grained heterogeneity on surface roughness is significant when there are fewer grains inside the specimen. When $\lambda$ is raised from 0.85 to $6.8, H_{s a}$ fall down from 0.025 to 0.004 dramatically. However, the variation of $H_{s a}$ is not so sensitive to the change of $\lambda$ when $\lambda$ exceeds 6.8, which means the influence of individual grain on the surface asperity get into a decline if a certain grain size is reached.

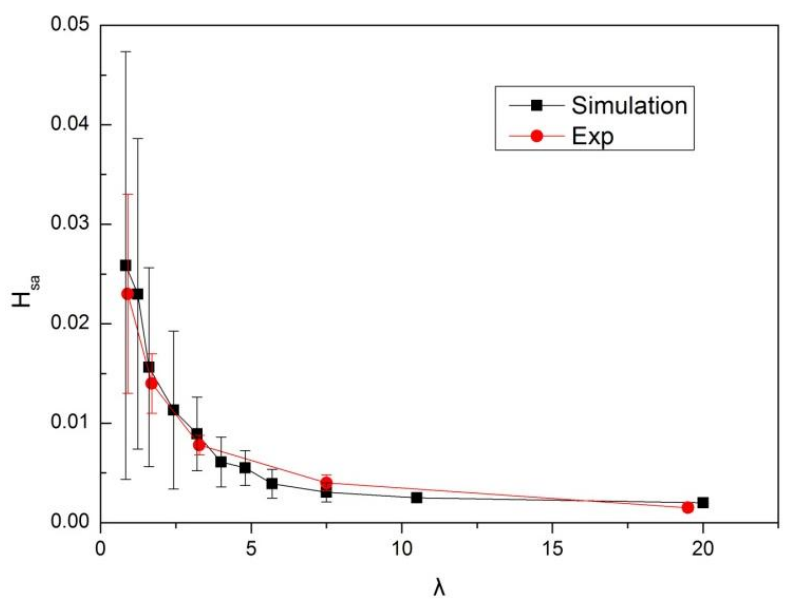

Figure 14. Influence of $\lambda$ on $\mathrm{H}_{\mathrm{sa}}$ 


\section{Application of the novel model in MCWR}

As shown in Fig. 15a, a FE model of MCWR (micro cross wedge rolling) process of pure cooper has been established taking into account the size effect using the novel model. It is used to predict and analyse the distribution of strain and stress and generation of defects. The geometrical dimension of the cylindrical specimen is $\Phi 0.8 \mathrm{~mm} \times 0.8 \mathrm{~mm}$ and the area reduction of deformed part is $43.75 \%$. The specimen model involves $1313 \times 40$ solid elements and other parameters are the same as that in micro compression. The equal and opposite velocities were applied on two wedges in $x$ direction, and the two wedges are fixed in both $y$ and $z$ directions.

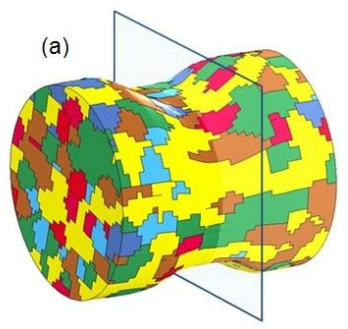

(b)

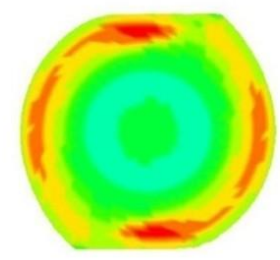

(c)

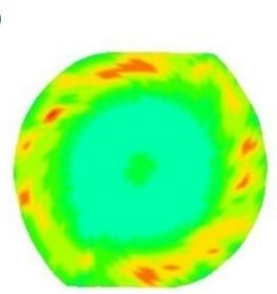

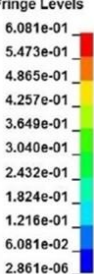

Figure 15. Distribution of effective strain of the medial section in axial direction (a) FE model of MCWR in proceeding (b) uniform material model (c) inhomogeneous material model

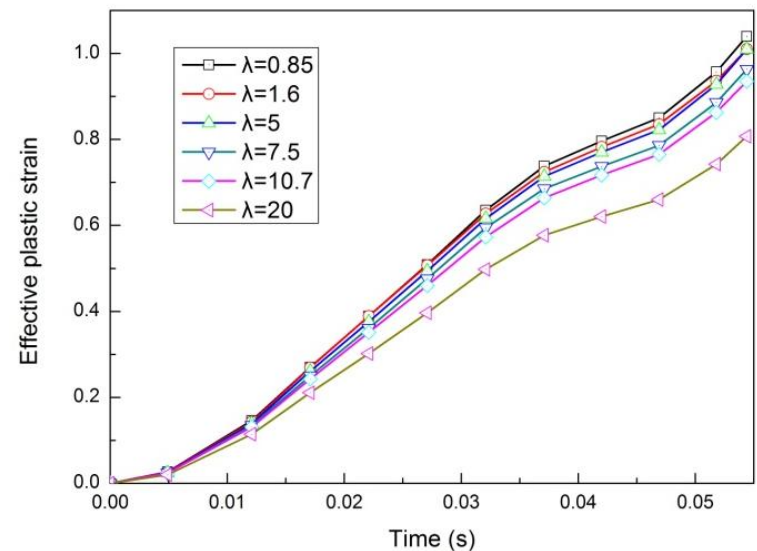

Figure 16. Effective strain in knifing and guiding zones

A characteristic of cross wedge rolling is the Laminar cricoid distribution of strain when the material properties are regarded as homogeneous. This phenomenon also occurs in MCWR if the material of billet is homogeneous (Fig. 15b). In micro scale, the influence of grained heterogeneity on the metal deformability and strain distribution cannot be neglected. The cross sections shown in Fig. 15 locate in the middle of the cylindrical specimen with $\lambda=3$. As shown in Fig. 15c, the continuous laminar distribution of strain in the workpiece has been disturbed due to the inhomogeneous mechanical properties. The location of maximum strain and stress cannot be determined easily as that in the CWR process without considering the size effects. Fig. 16 shows the curves of the effective strain at vicinity of specimen core region in knifing and guiding zones. The strain falls down with the increase of $\lambda$ value, which means there is more deformation around the core region of specimen with larger grain size.

\section{Conclusions}

A constitutive model considering grain boundary strengthening effect, surface layer model and grained heterogeneity has been proposed. A set of 3D aggregates of polycrystalline with different grain sizes was generated using voronoi tessellation. FEM simulation was conducted based on the 3D aggregate discretised by regular mesh to verify that constitutive model. Compression experiment was performed and the flow stress obtained from experiments shows little discrepancy compared with that of simulation. The scatter of grained deformation behaviour has been investigated through flow stress and side surface asperity of cylindrical specimen. The results show that the scatter of deformation behaviour becomes significant as the grain size increase while specimen size keeps constant. The novel material model is utilised to simulate MCWR process. The typical crocoid strain distribution in the middle section of the specimen is disturbed due to grained heterogeneity and the strain in the core region of the specimen decreases as the grain size increases. 


\section{Acknowledgements}

The authors would like to thank the Australia Research council (ARC) for its financial support.

\section{References}

[1] M. Geiger, M. Kleiner, R. Eckstein, N. Tiesler, U. Engle, Microforming, CIRP Manuf. Technol. 50 (2001) 445-462

[2] F. Vollertsen, H. Schulze Niehoff, Z. Hu, State of the art in micro forming, Int. J. Mach. Tools Manul. 46 (2006) 1172-1179.

[3] F. Vollertsen, D. Biermann, H.N. Hansen, I.s. Jawahir, K. Kuzman, Size effects in manufacturing of metallic components, CIRP Manuf. Technol.50 (2009) 566-587.

[4] M.W. Fu, Z.J. Luo, The prediction of macro-defects during the isothermal forging process by the rigid-viscoplastic Finite-Element Method, J. Mater. Proc.Technol.32 (1992) 559-608.

[5] E.O. Hall, Deformation and ageing of mild steel, Physical Society Proceeding 64 (1951) 747-753.

[6] N.J. Petch, Cleavage Strength of polycrystals, Iron and Steel Institute 174 (1953) 25-28.

[7] S. Miyazaki, K. Shibata, H, Fujita, Effect of specimen thickness on mechanical properties of polycrystalline aggregates with various grain sizes, Acta. Metall. 27 (1979) 855-862.

[8] D.K. Leu, Modeling of size effect on tensile flow stress of sheet metal in microforming, ASME J. Manuf. Sci. Eng. 131 (2009) 01100210110028.

[9] U. Engle, R. Eckstein, Microforming-from basic research to its realization, J. Mater. Proc. Tochnl. 125-126 (2002) 35-44

[10] R. Kals, H.J. Pucher, F. Volertesen, Effects of specimen size and Geometry in Metal Forming, In Hashmii SJ, (Ed.) Proceedings of the 2nd International Conference on Advances in Materials and Processing Technologies, 3 (1995) 1288-1297.

[11] X.M. Lai, L.F. Peng, P. Hu, S.H. Lan, J. Ni, Material behavior modelling in micro/meso-scale forming process with considering size/scale effects, Comput. Mterial. Sci. 43 (2008) 1003-1009.

[12] J.G. Liu, M.W. Fu, W.L. Chan, A constitutive model for modelling of the deformation behavior in microforming with a consideration of grain boundary strengthening, Comput. Mterial. Sci. 55 (2012) 85-94

[13] W.L. Chan, M.W. Fu, J. Lu, J.G. Liu, Modeling of grain size on micro deformation behaviour in micro-forming of pure copper, Material Science and Engineering A 527 (2010) 6638-6648.

[14] H.N. Lu, D.B. Wei, Z.Y. Jiang, K. Manabe, T. Furushima, Steel Research International, 2011,962-967.

[15] O. Diard, S. Leclercq, G. Rousselier, G. Cailetaud, Evaluation of finite element based analysis of 3D multicrystalline aggregates plasticity: Application to crystal plasticity model identification and the study of stress and strain fields near grain boundaries, Int. J. Plasticity 21 (2005) 691-722.

[16] A. G. Crocker, P. E. J. Flewitt and G. E. Smith, Computational Modeling of Fracture in Polycrystalline Materials, International Materials Reviews 50 (2005) 99-124.

[17] N. Zhang, W. Tong, An experimental study on grain deformation and interactions in an Al-0.5\%Mg multicrystal, Int. J. Plasticity 20 (2004) 523-542.

[18] B. Zhu, R.J. Asaro, P. Krysl, K. Zhang, J.R. Weertman, Effects of grain size distribution on the mechanical response of nanocrystalline metals: Part II, Acta Mater. 54 (2006) 3307-3320.

[19] F. Dalla Torre, H. Van Swygenhoven, M. Victoria, Nanocrystalline electrodeposited Ni: microstructure and tensile properties, Acta Mater. 50 (2002) 3957-3970.

[20] X.M. Lai, L.F. Peng, P. Liu, S.H. Lan, J. Ni, 2008. Material behavior modelling in micro/meso-scale forming process with considering size/scale effects, Comput. Mterial. Sci. 43(2008) 1003-1009.

[21] U.F. Kocks, Metal.Mater.Trans.B1, The relation between polycrystal deformation and single-crystal deformation, (1970) 1121-1143

[22] M.A. Meyers, E. Ashworth, A model for the effect of grain size on the yield stress of metals, Philos. Mg. 46 (1982) 737-759.

[23] H.H. Fu, D.J. Benson,M.a. Meyers, Analytical and computational description of effect of grain size on yield stress of metals, Acta Mater. 49 (2001) 2567-2582.

[24] M. Geiger, S. Geibdorfer, U. Engle, Mesoscopic model: advanced simulation of microforming processes, Production Engineering 1 (2007) 79-84

[25] S. Mahabunphachai, M. Koc, Investigation of size effects on material behavior of thin sheet metals using hydraulic bulge testing at micro/meso-scales, Int. J. Mach. Tools Manuf. 48 (2008) 1014-1029. 